Europhys. Lett., 59 (2), pp. 193-198 (2002)

\title{
Collective modes in parametrically excited oscillator arrays
}

\author{
D. Goldobin ${ }^{1,2}$ and A. PikovskY ${ }^{2}$
}

1 Department of Theoretical Physics, Perm State University

15 Bukireva str., 614990, Perm, Russia

2 Department of Physics, University of Potsdam

Postfach 601553, D-14415 Potsdam, Germany

(received 1 February 2002; accepted in final form 23 April 2002)

PACS. 05.45.-a - Nonlinear dynamics and nonlinear dynamical systems.

PACS. 89.75.Kd - Patterns.

\begin{abstract}
We consider a population of parametrically excited globally coupled oscillators in a weakly nonlinear state. The instabilities of collective modes lead to a traveling-wave regime, where intensities of oscillations of each oscillator vary periodically in time. For large excitation amplitudes a frozen state with nearly uniform oscillation intensities is observed.
\end{abstract}

Ensembles of coupled oscillators demonstrate extremely rich behaviors [1-3]. They appear in descriptions of Josephson junctions [4], multimode lasers [5], and charge density waves [6]. In the living world one uses similar models to describe chirps of grasshoppers [7], neurons [8-10] and yeast cells [11]. One of the most interesting effect in these ensembles is the appearance of collective modes, when at least a part of the oscillators is synchronized and the mean field exhibits nontrivial dynamics. Probably, the most impressive demonstration of this is a rhythmic flashing of fireflies [12]. In the theory, the onset of collective behavior is known as the Kuramoto transition [1], and it is a prominent example of nonequilibrium phase transitions.

Recently, populations of parametrically excited oscillators attracted particular interest [1316]. One possible realization of such a system is an array of Josephson junctions [17]; below we also describe a simple mechanical example of such an ensemble. In [13-15] a linear analysis of instabilities in the ensemble has been performed in a model where the parametric modulation is a piecewise function of time. Parametric excitation by a sinusoidal field in a linear chain of overdamped oscillators was described in [16].

In this paper we study instabilities and collective modes in globally coupled weakly nonlinear oscillators parametrically excited by a sinusoidal signal. Using the method of averaging, we obtain equations for slowly varying amplitudes. The analysis of these equations allows us to find linear instabilities as well as to analyse nonlinear modes developing from these instabilities. The analytical results are confirmed by numerical experiments.

As a prototypic model we consider a population of $N$ coupled weakly nonlinear oscillators subject to a parametric excitation. The governing equations are

$$
\ddot{x}_{i}+2 \gamma \dot{x}_{i}+\omega_{0}^{2}\left(1+\xi_{i}(t)\right) x_{i}+T\left(x_{i}, \dot{x}_{i}\right)=-\frac{\kappa}{N} \sum_{j=1}^{N}\left(x_{i}-x_{j}\right)-\frac{2 \sigma}{N} \sum_{j=1}^{N}\left(\dot{x}_{i}-\dot{x}_{j}\right) .
$$


Here terms $\sim \kappa$ and $\sim \sigma$ describe the conservative and dissipative coupling, respectively. The nonlinear terms $T\left(x_{i}, \dot{x}_{i}\right)$ will be specified below.

We assume the parameters of oscillators in the ensemble to be identical, but the parametric driving given by the term $\omega_{0}^{2} \xi_{i}(t) x_{i}$ has different phases for different oscillators: $\xi_{i}(t)=$ $2 A \cos \left(\omega_{p} t+\tau_{i}\right)$. The frequency of driving $\omega_{p}$ must be close to $2 \omega_{0}$ in order to achieve the strongest excitation, we take $\omega_{0}^{2}=\omega_{p}^{2}(1 / 4+\epsilon)$, where $\epsilon$ is a small mismatch. Below we assume that the phases of the driving $\tau_{i}$ are distributed uniformly on the interval $[0,2 \pi)$. Because there is no ordering of oscillators due to coupling, we can order them according to the phase of the driving force, assigning label $\tau$ instead of index $i$ to the variable $x$. Moreover, in the thermodynamic limit $N \rightarrow \infty$ we can replace the summation over $i$ with integration over $\tau$ :

$$
\frac{1}{N} \sum_{i=1}^{N} \rightarrow \frac{1}{2 \pi} \int_{0}^{2 \pi} \mathrm{d} \tau
$$

The main assumption for the theoretical analysis is that the oscillators are nearly harmonic, i.e. that the damping, forcing, coupling, and nonlinearity are small. In this case a standard averaging method (see, e.g., [18]) allows us to write equations for the slowly varying complex amplitudes $X_{\tau}(t)$, where $x_{\tau}(t)=\operatorname{Re}\left[\left(2 X_{\tau}(t) \exp \left[i \frac{\omega_{p}}{2} t\right]\right]\right.$ :

$$
\dot{X}_{\tau}=(-\gamma+i \epsilon) X_{\tau}+A e^{i \tau} X_{\tau}^{*}+(-\sigma+i \kappa)\left(X_{\tau}-\langle X\rangle\right)-(\alpha+i \beta) X_{\tau}\left|X_{\tau}\right|^{2} .
$$

Here the lowest-order nonlinear terms arising from the averaging of $T\left(x_{i}, \dot{x}_{i}\right)$ are written, in general both conservative $(\sim \beta)$ and dissipative $(\sim \alpha)$ nonlinearities are present.

Before proceeding in the analysis of eqs. (2) we show that such a system naturally appears in the description of a particular model of coupled mechanical oscillators. Let us consider an ensemble of $N$ mathematical pendula (having the same length $l$ and mass $m$ ) hanging on a movable plate of mass $M$. The plate moves in a horizontal direction (variable $y$ ) with a restoring force $-k y$, and its vertical bendings are organized according to a prescribed wavy mode whose displacement at the pivot of the pendulum $i$ is $\xi_{i}(t)=A l \cos \left(2 \omega t+\theta_{i}\right)$, where $\omega$ is close to the linear frequency of a pendulum $\sqrt{g / l}$. Denoting the angle of the $i$-th pendulum by $\phi_{i}$, we can write the Lagrangian

$$
L=\frac{M \dot{y}^{2}}{2}-\frac{k y^{2}}{2}+\sum_{i=1}^{N} \frac{m}{2}\left(\left(l \dot{\phi}_{i} \cos \phi_{i}+\dot{y}\right)^{2}+\left(l \dot{\phi}_{i} \sin \phi_{i}+\dot{\xi}_{i}\right)^{2}\right)-\sum_{i=1}^{N} m g\left(l-l \cos \phi_{i}+\xi_{i}\right) .
$$

The equations of motion, where we also introduce the damping terms $\sim R, r$, read

$$
\begin{aligned}
(M+N m) \ddot{y}+2 R \dot{y}+k y & =-m l \frac{\mathrm{d}^{2}}{\mathrm{~d} t^{2}} \sum_{i=1}^{N} \sin \phi_{i}, \\
\ddot{\phi}_{i}+2 r \dot{\phi}_{i}+\left(\frac{g+\ddot{\xi}_{i}}{l}\right) \sin \phi_{i} & =-\frac{\ddot{y}}{l} \cos \phi_{i} .
\end{aligned}
$$

Assuming the oscillations with slowly varying amplitude $\phi_{i}=\frac{1}{2}\left(a(t) e^{i \omega t}+a^{*}(t) e^{-i \omega t}\right), y=$ $\frac{1}{2}\left(B(t) e^{i \omega t}+B^{*}(t) e^{-i \omega t}\right)$ and neglecting nonlinearity in the coupling we obtain for each pendulum, applying the method of averaging, the equations of motion

$$
\dot{a}_{i}=-(r+i \Delta \omega) a_{i}-i \omega A_{i} a_{i}^{*}-i \frac{\omega}{16}\left|a_{i}\right|^{2} a_{i}-i \frac{\omega B}{2 l},
$$


where $A_{i}=A e^{i \theta_{i}}, \Delta \omega=\omega-(g / l)^{1 / 2}$. The amplitude $B$ can be expressed from the linearized equation for $y$ (it is required that this amplitude is small, and this condition should be checked for a given set of parameters):

$$
B=\frac{N m l \omega^{2}}{k-(M+N m) \omega^{2}+2 R i \omega} \cdot \frac{1}{N} \sum_{i=1}^{N} a_{i} .
$$

Substituting this in the equation for $a_{i}$, we obtain finally the globally coupled equations of motion in the form $(2)$

$$
\dot{a}_{i}=-(r+i \Delta \omega) a_{i}-i \omega A_{i} a_{i}^{*}-i \frac{\omega}{16}\left|a_{i}\right|^{2} a_{i}-i \frac{\omega^{3} N m}{2 k-2(M+N m) \omega^{2}+4 R i \omega}\left\langle a_{i}\right\rangle .
$$

In the investigation of linear instability it is convenient to rewrite eq. (2) in terms of "spatial" modes

$$
C_{k}=\frac{1}{2 \pi} \int_{0}^{2 \pi} e^{-i k \tau} X_{\tau} \mathrm{d} \tau
$$

Then, obviously, $\langle X\rangle=C_{0}$, and we obtain, neglecting the nonlinear terms,

$$
\dot{C}_{k}=(-\gamma+i \epsilon) C_{k}+A C_{1-k}^{*}+(-\sigma+i \kappa) C_{k}\left(1-\delta_{0, k}\right) .
$$

One can easily see that the whole system decomposes in pairs of coupled modes $k \leftrightarrow(1-k)$. Moreover, the equations for all pairs with $k \neq 0$ are the same, while for $k=0$ we have an extra term due to mean-field coupling. As a result we obtain from (3)

$$
\dot{C}_{0}=(-\gamma+i \epsilon) C_{0}+A C_{1}^{*}, \quad \dot{C}_{1}=(-(\gamma+\sigma)+i(\epsilon+\kappa)) C_{1}+A C_{0}^{*},
$$

for the pair $(0,1)$ and

$$
\dot{C}_{k}=(-(\gamma+\sigma)+i(\epsilon+\kappa)) C_{k}+A C_{1-k}^{*}, \quad \dot{C}_{1-k}=(-(\gamma+\sigma)+i(\epsilon+\kappa)) C_{1-k}+A C_{k}^{*},
$$

for all other pairs. Correspondingly, we obtain two regions of instability of the ground state $C=0$. The instability in eq. (4) is oscillatory and onsets for

$$
A^{2}>\left[1-\frac{\sigma^{2}}{(2 \gamma+\sigma)^{2}}\right]\left(\epsilon+\frac{\kappa}{2}\right)^{2}+\gamma(\gamma+\sigma)
$$

The linear system (5) has eigenvalues

$$
\lambda_{1,2}=-(\gamma+\sigma) \pm \sqrt{A^{2}-(\epsilon+\kappa)^{2}}
$$

and the monotonous (i.e. with real growth rate) instability onsets for

$$
A^{2}>(\gamma+\sigma)^{2}+(\epsilon+\kappa)^{2} .
$$

From the comparison of the two instability areas (fig. 1) one can see that the oscillatory instability in the modes $C_{0}, C_{1}$ usually onsets for smaller values of parametric forcing $A$, but for some frequency mismatches the instability of other modes $\left(C_{k}, C_{1-k}\right.$ with $\left.k \neq 0\right)$ onsets first. Note also that above we have distinguished the monotonous and the oscillatory instabilities on the base of the averaged equations (2), (3); in the original system (1) these 


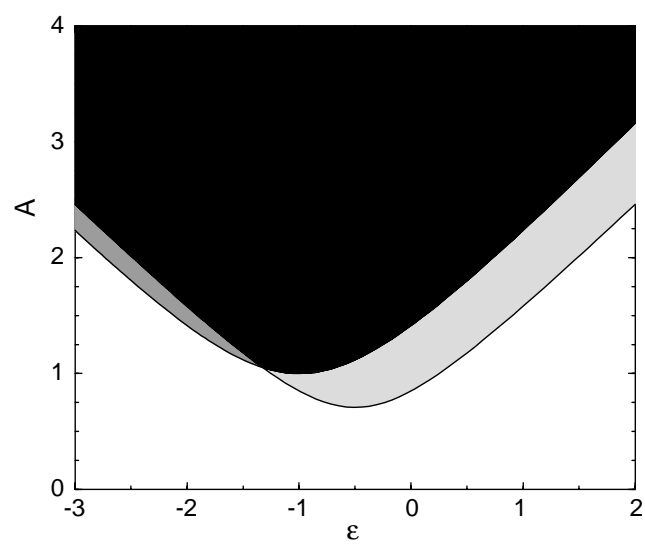

Fig. 1 - The instabilities areas for $\gamma=\sigma=1 / 2$ and $\kappa=1$. The dark-gray area is the region of monotonous instability, the light-gray is the region of oscillatory instability. In the black area both instabilities are present.

two types correspond to instabilities exactly at the half of the modulation frequency and at the shifted frequency.

In the next part of the paper we describe nonlinear regimes of parametric instability in the ensemble. We consider first the region on the plane of parameters $A, \epsilon$ where only the modes $C_{0}, C_{1}$ are unstable. We neglect the other stable modes and extract from (2) the nonlinear equations for the modes $C_{0}, C_{1}$ :

$$
\begin{array}{rr}
\dot{C}_{0}= & (-\gamma+i \epsilon) C_{0}+A C_{1}^{*}-(\alpha+i \beta)\left(\left|C_{0}\right|^{2}+2\left|C_{1}\right|^{2}\right) C_{0}, \\
\dot{C}_{1}= & (-\sigma-\gamma+i(\epsilon+\kappa)) C_{1}+A C_{0}^{*}-(\alpha+i \beta)\left(2\left|C_{0}\right|^{2}+\left|C_{1}\right|^{2}\right) C_{1} .
\end{array}
$$

The analysis of these equations significantly simplifies in the case $\sigma=0$, i.e. when the coupling between the oscillators is purely conservative. We restrict ourselves to this situation; furthermore we assume that $\gamma>0, \alpha>0$. Then it is easy to check that the following relation is valid:

$$
\frac{\mathrm{d}}{\mathrm{d} t}\left(\left|C_{0}\right|^{2}-\left|C_{1}\right|^{2}\right)=-\left(2 \gamma+2 \alpha\left(\left|C_{0}\right|^{2}+\left|C_{1}\right|^{2}\right)\right)\left(\left|C_{0}\right|^{2}-\left|C_{1}\right|^{2}\right) .
$$

This means that asymptotically for large times $\left|C_{0}\right|^{2}=\left|C_{1}\right|^{2}$. Considering the dynamics on this invariant manifold, we introduce the amplitude and the phases of the modes $C_{0}=$ $\rho \exp \left[i \frac{1}{2}(\theta-\phi)\right], C_{1}=\rho \exp \left[i \frac{1}{2}(\theta+\phi)\right]$ and obtain

$$
\begin{aligned}
& \dot{\rho}=-\gamma \rho+A \rho \cos \theta-3 \alpha \rho^{3}, \\
& \dot{\theta}=2 \epsilon+\kappa-2 A \sin \theta-6 \beta \rho^{2}, \\
& \dot{\phi}=\kappa .
\end{aligned}
$$

Equations (7), (8) constitute an autonomous two-dimensional system, where the phase space volume for $\gamma, \alpha>0$ is everywhere decreasing (this can be easily seen if one rewrites the equations in the variables $\rho \cos (\theta / 2), \rho \sin (\theta / 2))$. Thus, limit cycles are not possible, and the only attractors are stable fixed points. From $(7),(8)$ one can see that the trivial equilibrium $\rho=0$ becomes unstable for $A^{2}>\gamma^{2}+\left(\epsilon+\frac{\kappa}{2}\right)^{2}$, and for $\alpha \gamma>\beta\left(\epsilon+\frac{\kappa}{2}\right)$ the corresponding 
bifurcation is a supercritical pitchfork bifurcation. The stationary amplitude is

$$
\rho_{0}=\left(\frac{\left.\beta\left(\epsilon+\frac{\kappa}{2}\right)-\alpha \gamma+\sqrt{\left(\beta\left(\epsilon+\frac{\kappa}{2}\right)-\alpha \gamma\right)^{2}+\left(\alpha^{2}+\beta^{2}\right)\left(A^{2}-\gamma^{2}-\left(\epsilon+\frac{\kappa}{2}\right)^{2}\right.}\right)}{3\left(\alpha^{2}+\beta^{2}\right)}\right)^{1 / 2} .
$$

For $\alpha \gamma<\beta\left(\epsilon+\frac{\kappa}{2}\right)$ the bifurcation is subcritical, and a stable equilibrium exists already for $A>\left|\left(\epsilon+\frac{\kappa}{2}\right) \alpha+\gamma \beta\right|\left(\alpha^{2}+\beta^{2}\right)^{-1 / 2}$. Note that a stable equilibrium $\rho_{0}, \theta_{0}$ in the subsystem (7), (8) is combined with linearly growing phase $\phi$ according to (9). As a result, for the original slowly varying amplitudes of the oscillators, we obtain a solution

$$
X_{\tau}=\rho_{0} e^{i\left(\frac{\theta_{0}-\kappa t}{2}\right)}\left(1+e^{i(\kappa t+\tau)}\right) .
$$

The intensity of the oscillations

$$
\left|X_{\tau}\right|^{2}=2 \rho_{0}^{2}(1+\cos (\kappa t+\tau))
$$

is a wave propagating in the direction of decreasing $\tau$ with velocity $\kappa$. This collective mode is confirmed by numerical simulations (fig. 2).

Now we consider a situation when the modes $C_{0}, C_{1}$ are stable within (4), but other modes $C_{k}, C_{1-k}$ are unstable. Because the coupling is via the mean field $C_{0}$, in this case we essentially have an ensemble of uncoupled parametrically excited oscillators described by the equations

$$
\dot{X}_{\tau}=(-\gamma-\sigma+i(\epsilon+i \kappa)) X_{\tau}+A e^{i \tau} X_{\tau}^{*}-(\alpha+i \beta) X_{\tau}\left|X_{\tau}\right|^{2} .
$$
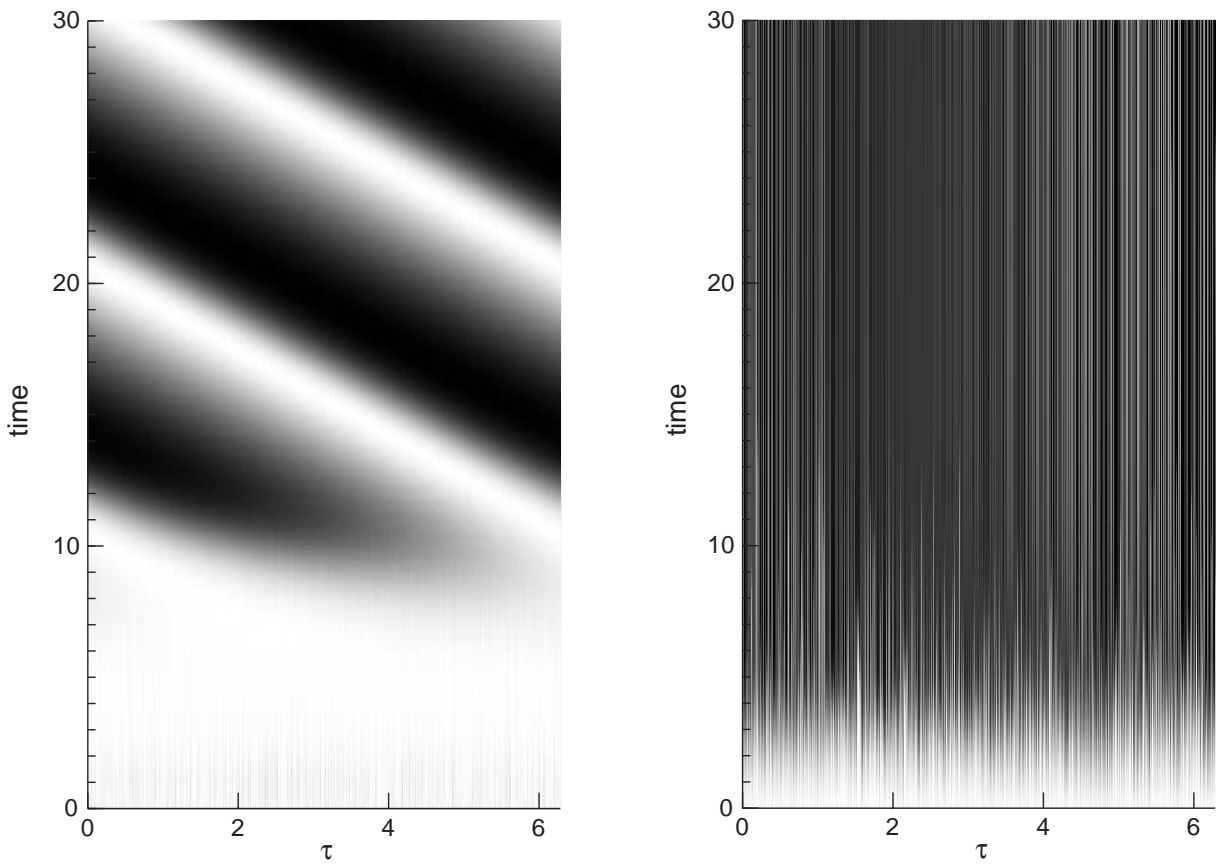

Fig. 2 - The results of the numerical simulation of system (2) for $\gamma=\sigma=\epsilon=\beta=1 / 2, \kappa=\alpha=1$, $N=1000$. The field intensity $\left|X_{\tau}\right|^{2}$ is shown by a gray scale. For $A=1.6$ (left panel) the propagating wave of type (10) develops from random initial conditions. For $A=2.2$ (right panel) all modes are unstable and a static field with relatively small mean field emerges. Because the mean field does not vanish, a small frozen modulation of the amplitude is observed. 
With the phase shift $X_{\tau} \rightarrow X_{\tau}^{\prime} e^{i \tau / 2}$ we can make all the equations equivalent. As a result, the amplitudes of oscillators are equal, and only the phases are different:

$$
X_{\tau}= \pm X^{(0)} e^{i \tau / 2} .
$$

Note that if such a field evolves from random initial conditions, than the signs in this solution are distributed randomly and the mean field does not vanish exactly but is small $(\sim 1 / \sqrt{N})$. Such a small field can be taken into account in (11) as a small constant perturbation. As a result, the amplitudes of oscillators are not equal, but are slightly statically modulated in $\tau$; additionally the two solutions for each $\tau$ corresponding to different signs in (12) are no more equal but slightly different. We illustrate such a "frozen" state in fig. 2 , and it can be observed also in the black area of fig. 1, where all modes are unstable. Near the border of black and light gray areas of fig. 1 we have observed multistability: depending on initial conditions we observed both the traveling wave and the frozen state.

In conclusion, we have described collective excitations in a population of parametrically excited oscillators. The instability threshold for a collective mode can lie below the instability threshold for single oscillators. In this case the two-mode weakly nonlinear description gives a traveling-wave solution, which is confirmed in the numerics. In this regime a single oscillator "fires" periodically in time, with the frequency proportional to the conservative part of the coupling.

$$
* * *
$$

DG thanks the DAAD Trilateral Partnership Program for support.

\section{REFERENCES}

[1] Kuramoto Y., Chemical Oscillations, Waves and Turbulence (Springer, Berlin) 1984.

[2] Pikovsky A., Rosenblum M. and Kurths J., Synchronization. A Universal Concept in Nonlinear Sciences (Cambridge University Press, Cambridge) 2001.

[3] Afraimovich V. S., Nekorkin V. I., Osipov G. V. and Shalfeev V. D., Stability, Structures and Chaos in Nonlinear Synchronization Networks (World Scientific, Singapore) 1994.

[4] Hadley P., Beasley M. R. and Wiesenfeld K., Phys. Rev. B, 38 (1988) 8712.

[5] Wiesenfeld K., Bracikowski C., James G. and Roy R., Phys. Rev. Lett., 65 (1990) 1749.

[6] Strogatz S. H., Marcus C. M., Westervelt R. M. and Mirollo R. E., Physica D, 36 (1989) 23.

[7] Sismondo E., Science, 249 (1990) 55.

[8] Stern E. A., Jaeger D. and Wilson C. J., Nature, 394 (1998) 475.

[9] Rulkov N. F., Phys. Rev. Lett., 86 (2001) 183.

[10] Tass P. A., Phase Resetting in Medicine and Biology. Stochastic Modelling and Data Analysis (Springer-Verlag, Berlin) 1999.

[11] Dano S., Sorensen P. G. and Hynne F., Nature, 402 (1999) 320.

[12] Buck J. and Buck E., Science, 159 (1968) 1319.

[13] Bena I. and Van den Broeck C., Europhys. Lett., 48 (1999) 498.

[14] Copelli M. and Lindenberg K., Phys. Rev. E, 63 (2001) 036605.

[15] Bena I. et al., Phys. Rev. E, 65 (2002) 036611.

[16] Farago J. and Van den Broeck C., Europhys. Lett., 54 (2001) 411.

[17] Nichols S. and Wiesenfeld K., Phys. Rev. E, 48 (1993) 2569.

[18] Bogoliubov N. N. and Mitropolsky Y. A., Asymptotic Methods in the Theory of Nonlinear Oscillations (Gordon and Breach, New York) 1961. 